\title{
A METRIZATION PROBLEM CONCERNING LATTICES
}

\section{H. J. CURTIS}

1. Introduction. The problem which we shall study stems from a paper by Wilcox [8]. He considered the problem of extending a metric topology of the point space of a semi-modular lattice to a topology for the entire lattice. This problem is of significance for the classical differential geometry of euclidean, affine, and projective spaces. Wilcox developed an extension of the topology of the point space in such a way that the lattice is a Hausdorff space. In doing this he assumed that the lattice is atomistic as well as semi-modular, that the elements of the lattice, considered as point sets, are closed in the topology of the point space, and that the greatest lower bound of distances from a given point to points of a nonzero element of the lattice is continuous in any set of independent points determining the nonzero element.

We consider the problem of determining conditions which are sufficient to insure that the lattice is metrizable. We show first that Wilcox's results are valid under slightly more general conditions than those which he assumed. Three additional axioms are introduced: first, the point space is separable; second, no element of dimension greater than one, considered as a point set, has arbitrarily small diameter; third, a generalization of Wilcox's axiom pertaining to the greatest lower bound of distances from a point to points of any nonzero element of the lattice.

We make use of a theorem of Urysohn to the effect that a separable metric space is homeomorphic to a subset of a compact subset of Hilbert space. If $P$ is the point space of a lattice $L$ which satisfies our axioms, we map $P$ topologically onto a subset $P^{\prime}$ of a compact subset of Hilbert space. A lattice $L^{\prime}$, isomorphic to $L$ and having $P^{\prime}$ as its point space, is easily constructed. Over $\overline{P^{\prime}}$, the closure of $P^{\prime}$, we construct a lattice $\overline{L^{\prime}}$ such that the elements of $\overline{P^{\prime}}$ are the points of $\overline{L^{\prime}}$, such that each element of $L^{\prime}$ is contained (as a point set) in an element of $\overline{L^{\prime}}$ and such that $\overline{L^{\prime}}$ satisfies the axioms of Wilcox. We can then extend the topology of $\overline{P^{\prime}}$ to $\overline{L^{\prime}}$ by the method of Wilcox. Since $\overline{P^{\prime}}$ is compact, we are able to show that $\overline{L^{\prime}}$ is compact. Then, using Wilcox's definition of neighborhood, we show that $\overline{L^{\prime}}$ has a countable

Presented to the Society, May 1, 1954; received by the editors November 12, 1954 and, in revised form, May 13, 1955. 
base. Hence, by another theorem of Urysohn, $\overline{L^{\prime}}$ is metrizable. Then $L^{\prime}$ is metrizable and consequently so is $L$. Nonisomorphic examples of lattices satisfying our axioms are the real euclidean and projective spaces. To show that our three axioms are not consequences of Wilcox's axioms, we give examples of lattices satisfying his axioms but not all of ours.

2. Foundations. Let $L$ be a lattice satisfying the ascending and descending chain conditions. There exist 0 and 1 in $L$ consequently, and every subset $S$ of $L$ has a meet, $\Sigma S$, and a join, $\pi S$, in $L$. We shall assume that $0 \neq 1$. Then there exists at least one $x \in L$ such that $x$ covers 0 (written $x>0$ ); i.e., $x>0$, and if $x>y \geqq 0$, then $y=0$. Let $P \equiv[p \in L ; p>0]$, and, for each $x \in L$, let $P_{x} \equiv[p \in P ; p \leqq x]$. We say that $L$ is atomistic if $x=\Sigma P_{x}$ for every $x \in L$. If $p \in P$, we call $p$ a point.

If $y, z \in L$, we call $(y, z)$ a modular pair, and write $(y, z) M$, provided that for every $x \leqq z,(x+y) z=x+y z$. A lattice $L$, satisfying both chain conditions, is called semi-modular if and only if, for every $x$, $y \in L, x+y>x, y$ whenever $x, y>x y$. Wilcox [6] has shown that $L$ is semi-modular if and only if $(x, y) M$ implies $(y, x) M$. If $L$ satisfies both the ascending and descending chain conditions, all chains in $L$ are finite. If $L$ is semi-modular and if $x \in L, x \neq 0$, the dimension of $x$, $d(x)$, is the largest positive integer $n$ such that there exist $x_{1}, \cdots$, $x_{n-1} \in L$ such that $0<x_{1}<\cdots<x_{n}=x$. That such a largest positive integer exists for each $x \neq 0$ has been proved by Birkhoff [2, p. 67]. We define $d(0)=0$ and let $d(1)=N$. Birkhoff $[2, \mathrm{pp} .67,100]$ has also proved that if $L$ is semi-modular, then $d(x)+d(y) \geqq d(x+y)+d(x y)$, the equality holding if and only if $(x, y) M$, and that $d(x)=d(y)+1$ if and only if $x>y$. Further it is evident that $d(x)>d(y)$ if $x>y$, and that $[d(x) ; x \in L]=[0,1, \cdots, N]$.

If $y, z \in L, y z=0$ and $(y, z) M$, we say that $y$ and $z$ are independent and write $(y, z) \perp$. We say that $x_{1}, \cdots, x_{n}$ are independent and write $\left(x_{1}, \cdots, x_{n}\right) \perp$, if $\Sigma\left(x_{i} ; i \in S\right)$ and $\Sigma\left(x_{j} ; j \in T\right)$ are independent, where $S$ and $T$ are subsets of $[1, \cdots, N]$ such that $i \in S$ and $j \in T$ implies that $i<j$. If $x=p_{1}+\cdots+p_{n}, p_{i} \in P$ and $\left(p_{1}, \cdots, p_{n}\right) \perp$, it is easily shown that $d(x)=n$.

If $P$ is a metric space with metric $\delta$, we define for every $x \in L$, $x \neq 0$, and every $p \in P$,

$$
\delta(p, x)=\delta(x, p)=\text { g.l.b. }[\delta(p, q) ; q \in P, q \leqq x] .
$$

Wilcox [8] has studied certain lattices which he called "semimodular" according to the following definition: a lattice $L$ satisfying the two chain conditions is "semi-modular" if $(x, y) \perp$ implies $(y, x) M$ 
and if $x y \neq 0$ implies $(x, y) M$, where $x, y \in L$. The second of these conditions is used only to establish the existence of the dimension function which we obtained above without the use of this condition. Consequently the results of Wilcox's paper are valid if we replace his definition of a semi-modular lattice by our definition. An example of a lattice in which the relation $M$ is symmetric but in which $x y \neq 0$ does not imply $(x, y) M$ has been given by Wilcox $[5$, p. 456].

We consider a lattice $L$ satisfying the following four axioms:

Axıом I. $L$ is semi-modular and atomistic.

Axıом II. $P$ is a metric space with metric $\delta$.

Aхіом III. Let $p, p_{i} \in P,\left(p_{1}, \cdots, p_{r}\right) \perp$. For every $\epsilon>0$ there exists $\gamma>0$ such that, if $\delta\left(p_{i}, q_{i}\right)<\gamma$, then $\mid \delta\left(p, p_{1}+\cdots+p_{n}\right)-\delta(p$, $\left.q_{1}+\cdots+q_{n}\right) \mid<\epsilon$.

Axiom IV. The sets $P_{x}$ are closed in the topology of $P$. These are the axioms of Wilcox except for the difference in the meaning of the word "semi-modular." In view of the remark of the preceding paragraph we know that it is possible to extend the topology of $P$ to $L$ in such a way that $L$ is a Hausdorff space. We shall show that under certain further assumptions $L$ is metrizable.

We include here several results pertaining to the relation $\perp$. The condition that $\left(p_{i}, p_{i+1}+\cdots+p_{n}\right) \perp$ for every $i=1, \cdots, n-1$ is necessary and sufficient that $\left(p_{1}, \cdots, p_{n}\right) \perp$ as has been proved by Wilcox $[7$, p. 505]. We easily obtain another necessary and sufficient condition that $\left(p_{1}, \cdots, p_{n}\right) \perp$, due originally to Menger $[3$, p. 462] for lattices satisfying Axiom I and an axiom of complementation.

Theorem 2.1. Let $p_{1}, \cdots, p_{n} \in P$. For every $n=2,3, \cdots$, $\left(p_{1}, \cdots, p_{n}\right) \perp$ if and only if $p_{i}\left(p_{i+1}+\cdots+p_{n}\right)=0$ for every $i=1, \cdots, n-1$.

Proof. The necessity is obvious in view of Wilcox's condition. The sufficiency follows from the semi-modularity of $L$.

Still another necessary and sufficient condition is given in terms of the metric $\delta$.

Theorem 2.2. Let $p_{1}, \cdots, p_{n} \in P$. For every $n=2,3, \cdots$, $\left(p_{1}, \cdots, p_{n}\right) \perp$ if and only if there exists $\phi>0$ such that $\delta\left(p_{i}, p_{i+1}\right.$ $\left.+\cdots+p_{n}\right)>\phi$ for every $i=1, \cdots, n-1$.

Proof. The theorem is a consequence of Axiom IV and Theorem 2.1.

We now introduce three additional axioms which we shall show are sufficient to establish the metrizability of $L$.

Aхıом V. $P$ is separable. 
Axiom VI. Let $\left\{p^{a}\right\},\left\{p_{i}^{a}\right\}$ be Cauchy sequences $(a=1,2, \cdots$ and $i=1, \cdots, n), p^{a}$ and $p_{i}^{a} \in P$. If there exists $\phi>0$ such that for every $a, \delta\left(p_{k}^{a}, p_{k+1}^{a}+\cdots+p_{n}^{a}\right)>\phi$ for every $k=1, \cdots, n-1$, then:

(a) $\lim _{a} \delta\left(p^{a}, p_{1}^{a}+\cdots+p_{n}^{a}\right)$ exists;

(b) there exists a Cauchy sequence $\left\{q^{a}\right\}, q^{a} \in P, q^{a} \leqq p_{1}^{a}+\cdots+p_{n}^{a}$, such that $\lim _{a} \delta\left(p^{a}, q^{a}\right)=\lim _{a} \delta\left(p^{a}, p_{1}^{a}+\cdots+p_{n}^{a}\right)$.

AxIom VII. There exists $\alpha>0$ such that for every set of independent points $p_{1}, \cdots, p_{n}$, there exist $q_{1}, \cdots, q_{n} \in P$ such that:

(a) $q_{1}+\cdots+q_{n}=p_{1}+\cdots+p_{n}$;

(b) $\delta\left(q_{k}, q_{k+1}+\cdots+q_{n}\right)>\alpha$ for every $k=1, \cdots, n-1$.

It is evident that Axiom VI is a generalization of Axiom III. That Axiom VI is not a consequence of Axioms I-IV will be shown in $\$ 6$ by an example of a lattice satisfying Axioms I-IV but not Axiom VI. Axiom VII rules out the possibility that any set $P_{x}$ has arbitrarily small diameter. We shall show in $\$ 6$ that it is independent of the other axioms.

3. Extension of $L$. A theorem due to Urysohn [4] states that a necessary and sufficient condition that a metric space $P$ be homeomorphic to a subset of a compact subset of Hilbert space is that $P$ be separable. We map $P$ topologically onto a subset $P^{\prime}$ of a compact subset of Hilbert space, and we let $\overline{P^{\prime}}$ be the closure of $P^{\prime}$ in this space. This mapping leads, by obvious definitions, to a lattice $L^{\prime}$, isomorphic to $L$, having $P^{\prime}$ as its point space and satisfying Axioms I-VII. We note that the metric for $P^{\prime}$ is not necessarily the usual metric for Hilbert space but merely an equivalent one. For simplicity of notation we shall henceforth refer to $P^{\prime}$ and $L^{\prime}$ as $P$ and $L$.

Our aim now is to extend $L$ to a lattice $\bar{L}$ which has $\bar{P}$ as its point space. We begin by extending the relation $\perp$ on $P$ cross $P$ to a relation $\perp^{*}$ on $\bar{P}$ cross $\bar{P}$. We prove several consequences of Axiom VI.

Lemma 3.1. Let $p \in \bar{P}$ and let $\left\{p_{i}^{a}\right\}$ be Cauchy sequences with $p_{i}^{a} \in P$ $(i=1, \cdots, n$ and $a=1,2, \cdots)$. If there exists $\phi>0$ such that $\delta\left(p_{k}^{a}, p_{k+1}^{a}+\cdots+p_{n}^{a}\right)>\phi$ for every $k=1, \cdots, n-1$, then:

(a) $\lim _{a} \delta\left(p, p_{1}^{a}+\cdots+p_{n}^{a}\right)$ exists;

(b) there exists a Cauchy sequence $\left\{q^{a}\right\}, q^{a} \in P, q^{a} \leqq p_{1}^{a}+\cdots+p_{n}^{a}$, such that $\lim _{a} \delta\left(p, q^{a}\right)$ is equal to $\lim _{a} \delta\left(p, p_{1}^{a}+\cdots+p_{n}^{a}\right)$.

Proof. Since $p \in \bar{P}$, there exists a Cauchy sequence $\left\{p^{a}\right\}, p^{a} \in P$, such that $\lim _{a} p^{a}=p$. By Axiom VI we know that $\lim _{a} \delta\left(p^{a}, p_{1}^{a}+\cdots\right.$ $\left.+p_{n}^{a}\right)$ exists; denote this limit by $A$. Also there exists a Cauchy sequence $\left\{q^{a}\right\}, q^{a} \in P, q^{a} \leqq p_{1}^{a}+\cdots+p_{n}^{a}$, such that $\lim _{a} \delta\left(p^{a}, q^{a}\right)=A$. Then it is clear that $\lim _{a} \delta\left(p, q^{a}\right)=A$. Now let $\epsilon>0$. For sufficiently 
large $a,\left|\delta\left(p^{a}, q^{a}\right)-A\right|<\epsilon$ and $\left|\delta\left(p^{a}, p_{1}^{a}+\cdots+p_{n}^{a}\right)-A\right|<\epsilon$ and $\delta\left(p, p^{a}\right)<\epsilon$. Therefore we see that

$$
\delta\left(p, p_{1}^{a}+\cdots+p_{n}^{a}\right) \leqq \delta\left(p, q^{a}\right) \leqq \delta\left(p, p^{a}\right)+\delta\left(p^{a}, q^{q}\right)<A+2 \epsilon .
$$

We also know that there exist $x^{a} \in P, x^{a} \leqq p_{1}^{a}+\cdots+p_{n}^{a}$, such that $\delta\left(p, x^{a}\right)<\delta\left(p, p_{1}^{a}+\cdots+p_{n}^{a}\right)+\epsilon$. Hence we have

$$
A<\delta\left(p, p_{1}^{a}+\cdots+p_{n}^{a}\right)+\epsilon \leqq \delta\left(p^{a}, x^{a}\right)+\epsilon
$$

and

$$
A<\delta\left(p^{a}, p\right)+\delta\left(p, x^{a}\right)+\epsilon<\delta\left(p, p_{1}^{a}+\cdots+p_{n}^{a}\right)+3 \epsilon .
$$

Thus it follows that $\lim _{a} \delta\left(p, p_{1}^{a}+\cdots+p_{n}^{a}\right)=A$.

THEOREM 3.1. Let $\left\{p_{i}^{a}\right\}$ and $\left\{q_{i}^{a}\right\}$ be Cauchy sequences with $p_{i}^{a}$ and $q_{i}^{a} \in P(i=1, \cdots, n$ and $a=1,2, \cdots)$, such that $\lim _{a} \delta\left(p_{i}^{a}, q_{i}^{a}\right)=0$. If there exist $\phi_{1}, \phi_{2}>0$ such that $\delta\left(p_{k}^{a}, p_{k+1}^{a}+\cdots+p_{n}^{a}\right)>\phi_{1}$ and $\delta\left(q_{k}^{a}, q_{k+1}^{a}+\cdots+q_{n}^{a}\right)>\phi_{2}$ for every $k=1, \cdots, n-1$, and if $\left\{x^{a}\right\}$ is a Cauchy sequence, $x^{a} \in P, x^{a} \leqq p_{1}^{a}+\cdots+p_{n}^{a}$, then there exist $y^{a} \in P$, $y^{a} \leqq q_{1}^{a}+\cdots+q_{n}^{a}$, such that $\lim _{a} \delta\left(x^{a}, y^{a}\right)=0$.

Proof. The theorem is a consequence of Lemma 3.1 and the compactness of $\bar{P}$.

Corollary. Let $x, p_{i} \in \bar{P}(i=1, \cdots, n)$ and let $p_{i}^{a}, q_{i}^{a}$ and $x_{i}^{a} \in P$ such that $\lim _{a} p_{i}^{a}=p_{i}, \lim _{a} q_{i}^{a}=q_{i}, \lim _{a} x^{a}=x$ and $x^{a} \leqq p_{1}^{a}+\cdots+p_{n}^{a}$. If there exist $\phi_{1}, \phi_{2}>0$ such that for every $a=1,2, \cdots, \delta\left(p_{k}^{a}, p_{k+1}^{a}+\cdots\right.$ $\left.+p_{n}^{a}\right)>\phi_{1}$ and $\delta\left(q_{k}^{a}, q_{k+1}^{a}+\cdots+q_{n}^{a}\right)>\phi_{2}$, then there exist $y^{a} \in P$, $y^{a} \leqq q_{1}^{a}+\cdots+q_{n}^{a}$, such that $\lim _{a} y^{a}=x$.

Definition 3.1. If $p_{1}, p_{2} \in \bar{P}$ and $p_{1} \neq p_{2}$, we say that $p_{1}$ and $p_{2}$ are independent and write $\left(p_{1}, p_{2}\right) \perp^{*}$.

Corollary. If $\left(p_{1}, p_{2}\right) \perp^{*}$, then $\left(p_{2}, p_{1}\right) \perp^{*}$.

Definition 3.2. If $p_{1}, p_{2} \in \bar{P}$ and $\left(p_{1}, p_{2}\right) \perp^{*}$, then $S\left(p_{1}, p_{2}\right) \equiv[s \in \bar{P}$; there exist sequences $\left\{p_{i}^{a}\right\}(i=1,2$ and $a=1,2, \cdots), p_{i}^{a} \in P$ and $\left(p_{1}^{a}, p_{2}^{a}\right) \perp$, such that $\lim p_{i}^{a}=p_{i}$, and there exist $s^{a} \in P, s^{a} \leqq p_{1}^{a}+p_{2}^{a}$, such that $\left.\lim _{a} s^{a}=s\right]$.

Corollary. $S\left(p_{1}, p_{2}\right)=S\left(p_{2}, p_{1}\right)$.

Definition 3.3. If $p_{i} \in \bar{P}(i=1, \cdots, n$ and $n=3,4, \cdots)$, we say that $p_{1}, \cdots, p_{n}$ are independent and write $\left(p_{1}, \cdots, p_{n}\right) \perp^{*}$, if $\left(p_{2}, \cdots, p_{n}\right) \perp^{*}$ and $p_{1}$ is not in $S\left(p_{2}, \cdots, p_{n}\right)$, where $S\left(p_{2}, \cdots, p_{n}\right)$ $\equiv\left[s \in \bar{P}\right.$; there exist sequences $\left\{p_{i}^{a}\right\}(i=2, \cdots, n$ and $a=1,2, \cdots)$, 
$p_{i}^{a} \in P,\left(p_{2}^{a}, \cdots, p_{n}^{a}\right) \perp$, such that $\lim _{a} p_{i}^{a}=p_{i}$, and there exist $s^{a} \in P$, $s^{a} \leqq p_{2}^{a}+\cdots+p_{n}^{a}$, such that $\left.\lim _{a} s^{a}=s\right]$.

We next show that $\left(p_{1}, \cdots, p_{n}\right) \perp^{*}$ is symmetric in the $p_{i}$ and hence that $S\left(p_{1}, \cdots, p_{n}\right)$ is also.

Theorem 3.2. If $\left(p_{1}, \cdots, p_{n}\right) \perp^{*}, p_{i} \in \bar{P}$, and if $\lim _{a} p_{i}^{a}=p_{i}, p_{i}^{a} \in P$, then there exists $\phi>0$ such that $\delta\left(p_{k}^{a}, p_{k+1}^{a}+\cdots+p_{n}^{a}\right)>\phi$ for every $k=1, \cdots, n-1$ and all sufficiently large $a(n=2,3, \cdots)$.

Proof. The theorem is easily proved by induction.

Theorem 3.3. If $p_{1}, \cdots, p_{n} \in \bar{P}, n \geqq 2$, and $\left(p_{1}, \cdots, p_{n}\right) \perp^{*}$ and if $T\left(p_{1}, \cdots, p_{n}\right)=\left[t \in \bar{P}\right.$; for every set of $n$ sequences $\left\{p_{i}^{a}\right\}, p_{i}^{a} \in P$, such that $\lim _{a} p_{i}^{a}=p_{i}$ and $\left(p_{1}^{a}, \cdots, p_{n}^{a}\right) \perp$, there exist $t^{a} \in P, t^{a} \leqq p_{1}^{a}+\cdots$ $+p_{n}^{a}$, such that $\left.\lim _{a} t^{a}=t\right]$, then $T\left(p_{1}, \cdots, p_{n}\right)=S\left(p_{1}, \cdots, p_{n}\right)$.

Proof. The theorem follows immediately from Theorems 2.2, 3.1 and 3.2.

Lemma 3.2. If $q \in S\left(p_{1}, \cdots, p_{n}\right), n \geqq 2, p_{i} \in \bar{P}$, and if $\left(q, p_{2}, \cdots\right.$, $\left.p_{n}\right) \perp^{*}$, then $S\left(q, p_{2}, \cdots, p_{n}\right)=S\left(p_{1}, \cdots, p_{n}\right)$.

Proof. It is easily shown by Theorem 3.3 that $S\left(p_{1}, \cdots, p_{n}\right)$ contains $S\left(q, p_{2}, \cdots, p_{n}\right)$. The reverse inclusion is established by Theorem 3.2 and the corollary to Theorem 3.1.

LEmma 3.3. If $\left(p_{1}, \cdots, p_{n}\right) \perp^{*}, p_{i} \in \bar{P}, n \geqq 2$, then $\left(p_{i_{1}}, \cdots, p_{i_{k}}\right) \perp^{*}$ for every $k=2, \cdots, n$ and $i_{1}<\cdots<i_{k}$.

Proof. This is a direct consequence of the definition of $\perp^{*}$.

Theorem 3.4. If $\left(p_{1}, \cdots, p_{n}\right) \perp^{*}, p_{i} \in \bar{P}, n \geqq 2$, then $\left(p_{i_{1}}, \cdots\right.$, $\left.p_{i_{n}}\right) \perp *$ for every permutation $i_{1}, \cdots, i_{n}$ of the integers $1, \cdots, n$.

Proof. Make an induction on $n$ and use Lemmas 3.2 and 3.3.

If $x \in L$ and $d(x)=n$, then any $n$ independent points in $P_{x}$ determine $x$; i.e., if $p_{i} \leqq x$ and $\left(p_{1}, \cdots, p_{n}\right) \perp$, then $x=p_{1}+\cdots+p_{n}$. We establish a similar property for the sets $S\left(p_{1}, \cdots, p_{n}\right)$.

Theorem 3.5. If $q_{1}, \cdots, q_{n} \in \bar{P}, q_{i} \in S\left(p_{1}, \cdots, p_{n}\right)$ and $\left(q_{1}, \cdots\right.$, $\left.q_{n}\right) \perp^{*}$, then $S\left(q_{1}, \cdots, q_{n}\right)=S\left(p_{1}, \cdots, p_{n}\right)$.

Proof. It is obvious that $S\left(q_{1}, \cdots, q_{n}\right)$ is contained in $S\left(p_{1}, \cdots\right.$, $\left.p_{n}\right)$. The reverse inclusion can be proved by the use of Theorem 3.2 and the corollary to Theorem 3.1.

In $L, p_{1}+\cdots+p_{N}=1$ if and only if $\left(p_{1}, \cdots, p_{N}\right) \perp$, where $p_{i} \in P$. Consequently $\left(p_{1}, \cdots, p_{n}\right) \perp$ is false if $n>N$. These facts have the following consequences for $\bar{L}$. 
Theorem 3.6. If $\left(p_{1}, \cdots, p_{N}\right) \perp$, then $S\left(p_{1}, \cdots, p_{N}\right)=\bar{P}$.

Corollary. If $p_{1}, \cdots, p_{N+1} \in \bar{P}$, then $\left(p_{1}, \cdots, p_{N+1}\right) \perp^{*}$ is false.

We are now ready to define $\bar{L}$, the extension of $L$. For simplicity in the statement of theorems we make the following definition.

Definition 3.4. If $p \in \bar{P}, S(p)=[p]$. We shall usually denote $S(p)$ simply by $p$.

DEFinition 3.5. Let $\bar{L}$ be the set consisting of the empty set and all sets of the form $S\left(p_{1}, \cdots, p_{n}\right)$, where $1 \leqq n \leqq N, p_{i} \in \bar{P}$, and $\left(p_{1}, \cdots, p_{n}\right) \perp^{*}$. We denote the empty set by $0^{*}$ and $\bar{P}$ by $1^{*}$.

Definition 3.6. If $X, Y \in \bar{L}, X \leqq{ }^{*} Y$ means $X \subset Y$.

Corollary. The sets $S(p), p \in \bar{P}$, are the "points" of $\bar{L}$.

It is obvious that $\bar{L}$ is partly ordered by $\leqq *$. The next theorem provides the basis for a definition of the meet of two elements of $\bar{L}$.

THEOREM 3.7. For every $n=1,2, \cdots$, if $p_{i} \in \bar{P}, i=1, \cdots, n$, there exist $k, 1 \leqq k \leqq n$, and $q_{1}, \cdots, q_{k} \in \bar{P}$, chosen from among the $p_{i}$, such that:

(a) $\left(q_{1}, \cdots, q_{k}\right) \perp^{*}$ and $p_{i} \in S\left(q_{1}, \cdots, q_{k}\right)$;

(b) if $k>1$ and if $r_{i} \in \bar{P}, i=1, \cdots, k-1$, such that $\left(r_{1}, \cdots\right.$, $\left.r_{k-1}\right) \perp^{*}$, then there exists $p_{j}$ such that $p_{j}$ is not in $S\left(r_{1}, \cdots, r_{k-1}\right)$;

(c) the set $S\left(q_{1}, \cdots, q_{k}\right)$ is unique.

Proof. If $p_{1}=\cdots=p_{n}$, we can take $q_{1}=p_{1}$. If two of the $p_{i}$ are distinct, say $p_{1}$ and $p_{2}$, then $\left(p_{1}, p_{2}\right) \perp^{*}$. If $p_{i} \in S\left(p_{1}, p_{2}\right), i=3, \cdots, n$, let $q_{1}=p_{1}$ and $q_{2}=p_{2}$. If $r \in \bar{P}$, it is clear that either $q_{1}$ or $q_{2} \neq r$; that is, either $p_{1}$ or $p_{2}$ is not in $S(r)$. If $p_{3}$ is not in $S\left(p_{1}, p_{2}\right)$, then $\left(p_{1}, p_{2}\right.$, $\left.p_{3}\right) \perp *$. Suppose $p_{i} \in S\left(p_{1}, p_{2}, p_{3}\right), i=4, \cdots, n$. Let $q_{i}=p_{i}$ for $i=1,2$, 3. If $\left(r_{1}, r_{2}\right) \perp^{*}$ and every $p_{i} \in S\left(r_{1}, r_{2}\right)$, then $S\left(p_{1}, p_{2}\right)=S\left(r_{1}, r_{2}\right)$ by Theorem 3.5. Hence $p_{3} \in S\left(p_{1}, p_{2}\right)$ contrary to $\left(p_{1}, p_{2}, p_{3}\right) \perp^{*}$. Therefore there exists $p_{j}$ not in $S\left(r_{1}, r_{2}\right)$.

We repeat this argument. By Theorems 3.5 and 3.6 we reach, in a finite number of steps, a set $S\left(q_{1}, \cdots, q_{k}\right), k \leqq n$, such that $q_{1}, \cdots, q_{k}$ satisfy conditions (a) and (b) and have been chosen from among the $p_{i}$. To show the uniqueness of $S\left(q_{1}, \cdots, q_{k}\right)$ suppose that $q_{1}^{\prime}, \cdots, q_{k^{\prime}}^{\prime}$ satisfy conditions (a) and (b). Then clearly $k^{\prime}=k$ by condition (b). Since the $q_{i}$ were chosen from among the $p_{j}$, every $q_{i} \in S\left(q_{1}^{\prime}, \cdots, q_{k}^{\prime}\right)$ and therefore, by Theorem $3.5, S\left(q_{1}^{\prime}, \cdots, q_{k}^{\prime}\right)=S\left(q_{1}, \cdots, q_{k}\right)$.

Definition 3.7. If $S\left(p_{1}, \cdots, p_{n}\right)$ and $S\left(t_{1}, \cdots, t_{m}\right)$ are in $\bar{L}$, then $S\left(p_{1}, \cdots, p_{n}\right) \oplus S\left(t_{1}, \cdots, t_{m}\right)$ is defined to be the unique set $S\left(q_{1}, \cdots, q_{k}\right)$, where $q_{1}, \cdots, q_{k}$ is any finite sequence which satis- 
fies the properties (a) and (b) of Theorem 3.7. We also define $0^{*} \oplus X$ $=X \oplus 0^{*}=X$ for every $X \in \bar{L}$.

Theorem 3.8. If $X, Y \in \bar{L}$ and $Z=X \oplus Y$, then $Z$ is the least upper bound of $X$ and $Y$.

The next theorem is preliminary to the definition of the join of two elements of $\bar{L}$.

Theorem 3.9. If $X=S\left(p_{1}, \cdots, p_{n}\right)$ and $Y=S\left(t_{1}, \cdots, t_{m}\right)$, then either $X \cap Y=0^{*}$ or there exists a unique $Z=S\left(q_{1}, \cdots, q_{k}\right), 1 \leqq k$ $\leqq \min [m, n]$, such that:

(a) $Z=X \cap Y$;

(b) for every $W \in \bar{L}$ such that $W \leqq * X$ and $W \leqq * Y$, it is true that $W \leqq * Z$.

Proof. The existence of $Z$ is established by the corollary to Theorem 3.6, its uniqueness by Theorem 3.5.

Definition 3.8. If $X$ and $Y \in \bar{L}$, then we define

(a) $X \odot Y \equiv Y \odot X \equiv 0^{*}$, if $X \cap Y=0^{*}$;

(b) if $X \cap Y \neq 0^{*}, X \odot Y=S\left(q_{1}, \cdots, q_{k}\right)$, where $q_{1}, \cdots, q_{k}$ is any finite sequence satisfying conditions (a) and (b) of Theorem 3.9.

Corollary. If $X$ and $Y \in \bar{L}$ and $Z=X \odot Y$, then $Z$ is the greatest lower bound of $X$ and $Y$.

In view of the preceding theorems and definitions the following theorem is clear.

Theorem 3.10. The system $\left(\bar{L}, \leqq^{*}, \oplus, \odot\right)$ is a lattice.

4. Properties of $\bar{L}$. It is now not difficult to show that $\bar{L}$ satisfies Axioms I-IV. We include only a proof that $\bar{L}$ satisfies Axiom III. Our method is to show that $\bar{L}$ satisfies Axiom VI, of which Axiom III is a consequence. The proof indicates the importance of Axiom VI to the extension of $L$ to $\bar{L}$.

Theorem 4.1. Let $\left\{p^{a}\right\},\left\{p_{i}^{a}\right\}$ be Cauchy sequences $(i=1, \cdots, n$ and $a=1,2, \cdots), p^{a}, p_{i}^{a} \in \bar{P}$. If there exists $\phi>0$ such that for every $a$, $\delta\left(p_{k}^{a}, p_{k+1}^{a} \oplus \cdots \oplus p_{n}^{a}\right)>\phi$ for every $k=1, \cdots, n-1$, then:

(a) $\lim _{a} \delta\left(p^{a}, p_{1}^{a} \oplus \cdots \oplus p_{n}^{a}\right)$ exists;

(b) there exists a Cauchy sequence $\left\{q^{a}\right\}, q^{a} \in \bar{P}, q^{a} \leqq p_{1}^{a} \oplus \cdots \oplus p_{n}^{a}$, such that $\lim _{a} \delta\left(p^{a}, q^{a}\right)=\lim _{a} \delta\left(p^{a}, p_{1}^{a} \oplus \cdots \oplus p_{n}^{a}\right)$.

Proof. For each $a$ there exist $p^{a b}, p_{i}^{a b} \in P$ such that $\lim _{b} p^{a b}=p^{a}$ and $\lim _{b} p_{i}^{a b}=p_{i}^{a}$. For every $a,\left(p_{1}^{a}, \cdots, p_{n}^{a}\right) \perp^{*}$, so there exist $\phi_{a}>0$ such that, for sufficiently large $b, \delta\left(p_{k}^{a b}, p_{k+1}^{a b}+\cdots+p_{n}^{a b}\right)>\phi_{a}$. Therefore, 
by Axiom VI, $\lim _{b} \delta\left(p^{a b}, p_{1}^{a b}+\cdots+p_{n}^{a b}\right)$ exists and there exists a Cauchy sequence $\left\{q^{a b}\right\}, q^{a b} \in P, q^{a b} \leqq p_{1}^{a b}+\cdots+p_{n}^{a b}$, such that $\lim _{b} \delta\left(p^{a b}, q^{a b}\right)=\lim _{b} \delta\left(p^{a b}, p_{1}^{a b}+\cdots+p_{n}^{a b}\right)$. It is clear that there exists $q^{a}=\lim _{b} q^{a b} \in \bar{P}$, and $q^{a} \leqq p_{1}^{a} \oplus \cdots \oplus p_{n}^{a}$. Then it follows that

$$
\begin{aligned}
\lim _{b} \delta\left(p^{a b}, q^{a b}\right) & =\delta\left(p^{a}, q^{a}\right) \\
& =\lim _{b} \delta\left(p^{a}, p_{1}^{a b}+\cdots+p_{n}^{a b}\right) \\
& =\delta\left(p^{a}, p_{1}^{a} \oplus \cdots \oplus p_{n}^{a}\right) .
\end{aligned}
$$

For every $\epsilon>0$ and every $a$, there exists $K_{a}>0$ such that $b>K_{a}$ implies that

$$
\left|\delta\left(p^{a}, p_{1}^{a b}+\cdots+p_{n}^{a b}\right)-\delta\left(p^{a}, p_{1}^{a} \oplus \cdots \oplus p_{n}^{a}\right)\right|<\epsilon .
$$

Let $b_{a}$ be the smallest integer greater than $K_{a}$. Then there exist $p_{i} \in \bar{P}$ such that $p_{i}=\lim _{a} p_{i}^{a}=\lim _{a} p_{i}^{a b}$, and, since there exists $\phi>0$ such that $\delta\left(p_{k}^{a}, p_{k+1}^{a} \oplus \cdots \oplus p_{n}^{a}\right)>\phi$ for every $k=1, \cdots, n-1,\left(p_{1}, \cdots\right.$, $\left.p_{n}\right) \perp^{*}$. Therefore there exists $\gamma>0$ such that $\delta\left(p_{k}^{a b_{a}}, p_{k+1}^{a b_{a}}+\cdots+p_{n}^{a b_{a}}\right)$ $>\gamma$ for every $k=1, \cdots, n-1$ and for sufficiently large $a$. By Axiom VI we know that $\lim _{a} \delta\left(p^{a b_{a}}, p_{1}^{a b_{a}}+\cdots+p_{n}^{a b_{a}}\right)$ exists and that there also exists a Cauchy sequence $\left\{r^{a b_{a}}\right\}, r^{a b_{a}} \in P, r^{a b_{a}} \leqq p_{1}^{a b_{a}}+\cdots+p_{n}^{a b_{a}}$, such that $\lim _{a} \delta\left(p^{a b_{a}}, r^{a b_{a}}\right)=\lim _{a} \delta\left(p^{a b_{a}}, p_{1}^{a b_{a}}+\cdots+p_{n}^{a b_{a}}\right)$. Since $\bar{P}$ is compact, there exists $p \in \bar{P}$ such that $p=\lim _{a} p^{a}=\lim _{a} p^{a b_{a}}$, and there exists $r \in \bar{P}$ such that $r=\lim _{a} r^{a b_{a}}$ and $r \leqq p_{1} \oplus \cdots \oplus p_{n}$. Therefore we have:

$$
\begin{aligned}
\lim _{a} \delta\left(p^{a b_{a}}, r^{a b_{a}}\right) & =\lim _{a} \delta\left(p^{a b_{a}}, p_{1}^{a b_{a}}+\cdots+p_{n}^{a b a}\right)=\delta(p, r) \\
& =\lim _{a} \delta\left(p^{a}, p_{1}^{a b_{a}}+\cdots+p_{n}^{a b_{a}}\right) \\
& =\lim _{a} \delta\left(p^{a}, p_{1} \oplus \cdots \oplus p_{n}\right) \\
& =\delta\left(p, p_{1} \oplus \cdots \oplus p_{n}\right) .
\end{aligned}
$$

For sufficiently large $a$

$$
\left|\delta\left(p^{a}, p_{1}^{a b_{a}}+\cdots+p_{n}^{a b q}\right)-\delta\left(p^{a}, p_{1} \oplus \cdots \oplus p_{n}\right)\right|<\epsilon .
$$

Since we also have

$$
\left|\delta\left(p^{a}, p_{1}^{a} \oplus \cdots \oplus p_{n}^{a}\right)-\delta\left(p^{a}, p_{1}^{a b a}+\cdots+p_{n}^{a b a}\right)\right|<\epsilon,
$$

we conclude that 


$$
\begin{gathered}
\left|\delta\left(p^{a}, p_{1}^{a} \oplus \cdots \oplus p_{n}^{a}\right)-\delta\left(p^{a}, p_{1} \oplus \cdots \oplus p_{n}\right)\right|<2 \epsilon, \\
\left|\delta\left(p^{a}, p_{1}^{a} \oplus \cdots \oplus p_{n}^{a}\right)-\delta\left(p, p_{1} \oplus \cdots \oplus p_{n}\right)\right|<2 \epsilon .
\end{gathered}
$$

Therefore $\lim _{a} \delta\left(p^{a}, p_{1}^{a} \oplus \cdots \oplus p_{n}^{a}\right)$ exists and equals $\delta\left(p, p_{1} \oplus \cdots\right.$ $\left.\oplus p_{n}\right)$. Since $\delta\left(p^{a}, q^{a}\right)=\delta\left(p^{a}, p_{1}^{a} \oplus \cdots \oplus p_{n}^{a}\right)$ and since $\lim _{a} \delta\left(p^{a}\right.$, $\left.p_{1}^{a} \oplus \cdots \oplus p_{n}^{a}\right)$ exists, it follows that $\lim _{a} \delta\left(p^{a}, q^{a}\right)=\lim _{a} \delta\left(p^{a}\right.$, $\left.p_{1}^{a} \oplus \cdots \oplus p_{n}^{a}\right)=\delta\left(p, p_{1} \oplus \cdots \oplus p_{n}\right)$. Finally, we see that $\lim _{a} \delta\left(p^{a}\right.$, $\left.q^{a}\right)=\lim _{a} \delta\left(p, q^{a}\right)$ implies that $\left\{q^{a}\right\}$ is a Cauchy sequence.

5. Compactness of $\bar{L}$ and the metrization of $L$. Since $\bar{L}$ satisfies Axioms I-IV, the topology of $\bar{P}$ can be extended to $\bar{L}$ in the same manner in which Wilcox extended the topology of $P$ to $L$. With respect to this topology $\bar{L}$ is a Hausdorff space [8, p. 283]. We can show further that $\bar{L}$ is compact relative to this topology. Urysohn $[1$, p. 82] has shown that a compact space is metrizable if and only if it has a countable base. We show that $\bar{L}$ has a countable base and hence that $\bar{L}$ is metrizable. The metrization of $\bar{L}$ provides the desired metrization of $L$.

THEOREM 5.1. If $P$ is compact, then $L$ is compact.

Proof. Let $\left\{x^{a}\right\}$ be a sequence with $x^{a} \in L$. Since $d(1)$ is finite, $\left\{x^{a}\right\}$ has at least one subsequence consisting entirely of elements of the same dimension. If this dimension is 0,1 or $N$, it is obvious that the subsequence has a limit in $L$. Otherwise, for simplicity of notation, let us assume that $d\left(x^{a}\right)=n, 2 \leqq n<N$, for every $a$. For each $x^{a}$ let $x^{a}=p_{1}^{a}+\cdots+p_{n}^{a}, p_{i}^{a} \in P$. Note that $\left(p_{1}^{a}, \cdots, p_{n}^{a}\right) \perp$. Since $P$ is compact, there exists a subsequence $\left\{a_{b}\right\}$ of $\{a\}$ such that $\lim _{b} p_{i}^{a_{b}}$ exists for each $i=1, \cdots, n$. Let $p_{i} \equiv \lim _{b} p_{i}^{a_{b}}$. Clearly $p_{1}^{a_{b}}+\cdots+p_{n}^{a_{b}}$ $=x^{a b}$ and $\left(p_{1}^{a_{b}}, \cdots, p_{n}^{a_{b}}\right) \perp$. Therefore, by Axiom VII, we can replace the $p_{i}^{a_{b}}$, for every $b$, by points $q_{i}^{a_{b}}$ such that $q_{1}^{a_{b}}+\cdots+q_{n}^{a_{b}}=p_{1}^{a_{b}}+\cdots$ $+p_{n}^{a_{b}}$ and such that $\delta\left(q_{k}^{a_{b}}, q_{k+1}^{a_{b}}+\cdots+q_{n}^{a_{b}}\right)>\alpha>0$ for every $k=1, \cdots, n-1$, where $\alpha$ is independent of the $p_{i}^{a_{b}}$. Again since $P$ is compact, there exists a subsequence of $\left\{a_{b}\right\}$, which we denote for simplicity as $\{c\}$, such that $\lim _{c} q_{i}^{c}$ exists. Let $q_{i} \equiv \lim _{c} q_{i}^{c}$.

We now show that $\left(q_{1}, \cdots, q_{n}\right) \perp$. If $q_{n-1}=q_{n}$, then $\delta\left(q_{n-1}^{c}, q_{n}^{c}\right)$ can be made arbitrarily small, contrary to $\delta\left(q_{n-1}^{c}, q_{n}^{c}\right)>\alpha$. Therefore, if $\left(q_{1}, \cdots, q_{n}\right) \perp$ is false, there exists a minimum $m, 1<m \leqq n-1$, such that $\left(q_{m}, \cdots, q_{n}\right) \perp$, while $\left(q_{m-1}, \cdots, q_{n}\right) \perp$ is false. Then $q_{m-1}\left(q_{m}+\cdots+q_{n}\right) \neq 0$ and $q_{m-1} \leqq q_{m}+\cdots+q_{n}$. Under these conditions Wilcox $\left[8\right.$, pp. 278-279] has shown that $\lim _{c}\left(q_{m}^{c}+\cdots+q_{n}^{c}\right)$ $=q_{m}+\cdots+q_{n}$, and also that there exist $x^{c} \in P, x^{c} \leqq q_{m}^{c}+\cdots+q_{n}^{c}$, such that $\lim _{c} x^{c}=q_{m-1}$. But $\lim _{c} q_{m-1}^{c}=q_{m-1}$, so $\lim _{c} \delta\left(x^{c}, q_{m-1}^{c}\right)=0$. 
Therefore $\lim _{c} \delta\left(q_{m-1}^{c}, q_{m}^{c}+\cdots+q_{n}^{c}\right)=0$, contrary to the fact that $\delta\left(q_{m-1}^{c}, q_{m}^{c}+\cdots+q_{n}^{c}\right)>\alpha$ for every $c$. Finally, since $\left(q_{1}, \cdots, q_{n}\right) \perp$ is now known to be true, we can apply a theorem of Wilcox [8, p. 279, Theorem 2.3], according to which $\lim _{c}\left(q_{1}^{c}+\cdots+q_{n}^{c}\right)=q_{1}+\cdots+q_{n}$. Therefore $L$ is compact.

In order to show that $\bar{L}$ is compact we need merely to verify that $\bar{L}$ satisfies Axiom VII. Then since $\bar{P}$ is compact, we can repeat the proof of Theorem 5.1. The verification depends upon the compactness of $\bar{P}$, Axiom VII (applied to $L$ ), and Theorem 3.5.

\section{THEOREM 5.2. $\bar{L}$ has a countable base.}

Proof. Let $p^{1}, p^{2}, p^{3}, \cdots$ be dense in $P$ (and hence in $\bar{P}$ ). A countable base for $\bar{L}$ is obtained by taking the set of all neighborhoods of the form $U\left(x ; 1 / k ; p^{a_{1}}, \cdots, p^{a_{n}}\right)$, where $x=p^{a_{1}}+\cdots+p^{a_{n}}$, $\left[a_{1}, \cdots, a_{n}\right] \subset[1, \cdots, N], k=1,2, \cdots$, defined by Wilcox $[8$, p. 281] as the set of $y \in \bar{L}$ with $d(y)=n$ such that $\delta\left(p^{a_{i}}, y\right)<1 / k$. To this set of neighborhoods we adjoin the empty set. That this set is a base for $\bar{L}$ can be established with the help of lemmas of Wilcox [8, p. 282, Lemmas 3.3 and 3.4].

We have thus proved that $\bar{L}$ is metrizable, in view of Urysohn's theorem.

\section{TheOREM 5.3. $L$ is metrizable.}

6. Independence of the axioms. Axioms I-VII are satisfied by the real euclidean plane and also by the real projective plane. In the former plane we may take $\delta$ to be the ordinary euclidean metric and in the latter plane to be the elliptic metric. We note that the planes are not isomorphic.

We now give an example of a space satisfying Axioms I-VI but not Axiom VII. Consider all points inside and on the boundary of a triangle in the euclidean plane. Let the lines of the space be determined as in the full plane and let the metric for the point space be the euclidean metric. Obviously Axiom VII is not satisfied, since there exist lines which, considered as point sets, have arbitrarily small diameter.

The following space satisfies Axioms $\mathrm{I}-\mathrm{V}$ and VII, but does not satisfy Axiom VI. Let $P$ be the set of points in the euclidean plane consisting of: (a) the points $(1,1 / n)$ and $(-1,1 / n)$ for $n=1,2, \cdots$; (b) the points $\left(0,1 / 2^{n}\right)$ for $n=0,1, \cdots$. Let the point metric be the euclidean metric, and for each pair of points $p_{1}, p_{2}$, let $p_{1}+p_{2}$ be the intersection of the line containing $p_{1}$ and $p_{2}$ in the full plane and $P$. It is clear that Axioms $\mathrm{I}-\mathrm{V}$ and VII are satisfied. To show that 
Axiom VI is not satisfied let $p_{1}^{a}=(-1,1 / a)$ and $p_{2}^{a}=(1,1 / a)$ and $p^{a}=(0,1)$, for every $a$. Then $\lim _{a} \delta\left(p^{a}, p_{1}^{a}+p_{2}^{a}\right)$ does not exist, because $\delta\left(p^{a}, p_{1}^{a}+p_{2}^{a}\right)=1-1 / a$, if $a=2^{k}, k=0,1, \cdots$, while $\delta\left(p^{a}, p_{1}^{a}+p_{2}^{a}\right)$ $=\left(1+\left(1-1 / a^{2}\right)\right)^{1 / 2}$, if $a \neq 2^{k}$. In the former case the limit is 1 , while in the latter it is $2^{1 / 2}$.

7. Conclusion. We mention here some questions raised by our study. The most important problem is to find a method of metrization which is not dependent upon the theory of compact spaces. We note that Axioms V and VII are directly involved in the use of the theory of compact spaces. Both axioms were introduced in order to make available the results of Urysohn's theorems. The motivation for the introduction of Axiom VI is perhaps less obvious. The primary purpose was to extend the property of $L$ given by Axiom III to $\bar{L}$. Axiom VI was also used to prove that $\left(p_{1}, \cdots, p_{n}\right) \perp^{*}$ is symmetric in the $p_{i}$. Since Axiom VI plays an important role in the extension of $L$ to $\bar{L}$, it is ultimately directed toward the use of the theory of compact spaces. A sufficient condition that a lattice satisfying Axioms I-IV be metrizable is that it satisfy Axioms V-VII. The question of necessary conditions remains open. That Axiom $V$ is not a necessary condition is known by an example. Whether there are any lattices which satisfy Axioms I-IV but are not metrizable is not known.

\section{BiBLIOGRAPHY}

1. P. Alexandroff and P. Urysohn, Memoire sur les espaces topologiques compacts' Verhandelingen der Koninklijke Nederlandsche Akademie van Wetenschappen, Afdeeling Natuurkunde, vol. XIV, no. 1, Amsterdam, 1929.

2. G. Birkhoff, Lattice theory, rev. ed., Amer. Math. Soc. Colloquium Publications, New York, 1948.

3. K. Menger, New foundations of projective and affine geometry, Ann. of Math. vol. 37 (1936) pp. 456-482.

4. P. Urysohn, Der Hilbertsche Raum als Urbild der metrischen Raume, Math. Ann. vol. 92 (1923) pp. 302-304.

5. L. R. Wilcox, $A$ note on complementation in lattices, Bull. Amer. Math. Soc. vol. 48 (1943) pp. $453-457$.

6. - Modularity in Birkhoff lattices, Bull. Amer. Math. Soc. vol. 50 (1944) pp. $135-138$. 505.

7. - Modularity in the theory of lattices, Ann. of Math. vol. 40 (1939) pp. 490-

8. - A topology for semi-modular lattices, Duke Math. J. vol. 8 (1941) pp. 273-285.

University of Illinois, Chicago 